\title{
Embedding net zero in health and care for the good of our health and our health service
}

\author{
Elaine Mulcahy director UK Health Alliance on Climate Change
}

Health and care services exist to improve wellbeing through interventions that seek to prevent ill health, protect from harm, and provide care at the point of need.

With increasing numbers of people living with multiple health conditions, the growing threat of urgent and prolonged ill health due to climate change, growing levels of health inequalities, and a health workforce stretched beyond capacity, it is vital that the health service we turn to when we're in need, is not one that is contributing to the problem.

The Health and Care Bill currently going through UK parliament provides an important moment to shift the delivery model of the health service in England to one that operates on a foundation of collaboration and integration.

The key purpose of the bill is to formulate the integration of healthcare systems in NHS England by legislating for every part of England to be covered by an Integrated Care Board (ICB) and Integrated Care Partnership (ICP) that ultimately seeks to support and enable collaboration between the NHS, local authorities, voluntary and community sectors, and other stakeholders.

The way in which these measures are implemented in practice have the potential to address many of the significant challenges currently facing our health and our health service, including chronic workforce shortages, increasing levels of health inequalities, and the devastating impact of climate change on health-and importantly seeking to ensure the health service is not contributing to that ill health.

In its current form, the bill does not include commitments to deliver on the UK's climate change and environment goals or a commitment to achieve net zero emissions targets. Omitting sustainability requirements is a missed opportunity to incorporate a drive to deliver services that not only seek to better integrate different aspects of care, but to do so in a manner that is environmentally responsible.

Climate change is currently the greatest threat to human health. In the last year, health professionals have made multiple calls for climate change mitigation and adaptation actions to protect public health. This has included an editorial simultaneously published across 220+international health journals and the delivery of a letter holding the signatures of organisations and individuals representing about 45 million health workers to world leaders attending COP26. ${ }^{12}$

The health burden of climate change is happening now, with rising global temperatures and air pollution contributing to the direct and immediate increase in major diseases including asthma, heart disease, and cancer. In the UK, exposure to air pollution is already causing up to 36 ooo deaths annually and disproportionately affects the most deprived areas of the country, further exacerbating health inequalities and stretching the health workforce. ${ }^{3}$

While working hard to care for those whose health is impacted by the effects of climate change, the healthcare sector is also a major contributing factor to the UK's carbon emissions. The NHS is responsible for about 4-5\% of England's annual emissions and $40 \%$ of all public sector emissions. This is comparable to the total annual emissions of countries such as Denmark or Croatia.

Since 2010, the NHS has reduced its emissions by $30 \%$ with interventions to improve patient care, increase efficiency and save lives. Recent data suggest that 9 in 10 staff of the 1.4 million people who work in the NHS support the NHS taking further action on climate change.

In October 2020, the NHS England and NHS Improvement became the world's first national health service to agree net zero commitments, setting out an ambitious path to reduce the NHS carbon footprint to net zero by 2040, and to reduce the total carbon footprint, including the international supply chain, to net zero by 2045 . Significant progress has already been made including policies to switch to less environmentally harmful inhalers and anaesthetic gases, investments in renewable energy and decarbonisation schemes, a net zero supplier roadmap, and the world's first zero emission ambulance. The national strategy is also being supported by many local initiatives that are being implemented across the country.

While the NHS has made huge progress on the path to net zero, the Health and Care Bill provides an opportunity to cement this ambition and embed the importance of net zero healthcare within legislation.

With the support of leaders from across the healthcare profession, the UK Health Alliance on Climate Change recently wrote to the Secretary of State for Health and Care calling for the role the NHS needs to play to deliver the UK's climate change and environment goals to be strengthened in law in order to integrate an active response to climate change through every layer of the NHS, including the new Integrated Care Boards. ${ }^{4}$

The climate crisis is already resulting in negative health impacts as a consequence of extreme weather, polluted air, food and water shortages, forced migration, and the aggravation of disease. We must do all we can to ensure the health and care services that people turn to during ill health, is not one of the 


\section{OPINION}

major contributing factors to that ill health. By embedding commitments to net zero in the Health and Care Bill the UK can lead the way in achieving this.

Competing interests: none declared

Provenance and peer review: commissioned, not peer reviewed

1 Atwoli L, Baqui AH, Benfield T, etal. Call for emergency action to limit global temperature increases, restore biodiversity, and protect health. BMJ2021;374:n1734

doi: 10.1136/bmj.n1734. pmid: 34483099

2 Healthy climate prescription. https://healthyclimateletter.net/

3 Public Health England. Review of interventions to improve outdoor air quality and public health. June 2020. https://assets.publishing.service.gov.uk/government/uploads/system/uploads/attachment_data/file/937341/PrincipaL_interventions_for_local_authorities-air_quality_public_health.pdf

4 UK Health Alliance on Climate Change. UK Alliance calls for Net Zero commitments in Health and Care Bill. http://www.ukhealthalliance.org/uk-health-alliance-calls-for-net-zero-commitments-inhealth-and-care-bill/ 